Hi Spirits

Why this matters

\section{User-generated content (UGC)}

UGC is outside the remit of the CAP Code, unless a marketer incorporates the content into a marketing communication, directly connecting it with the supply of goods or services. The ASA applies two tests when deciding whether UGC should be treated as an advertisement:

(i) has the marketer incorporated the content into their own marketing communications; and

(ii) has the marketer promoted the UGC?

Hi Spirits posted a user-generated image featuring a young man draped in a flag bearing the brand's logo on their Facebook page along with their own wording. The use of the image with their own wording amounted to incorporating the content into their marketing communications, and they actively promoted it by posting it on Facebook. Therefore, the ASA concluded that this amounted to a marketing communication on behalf of Hi Spirits, as its main purpose was to sell their product.

Advertisers should use this Guidance to check that communications appearing on their own websites or in non-paid-for space online under their control could be viewed by the ASA as a marketing communication. If they establish, using the tests described above, that their communication falls within the ASA's remit, advertisers should ensure that it complies with the CAP Code.

Fiona Schneider, trainee solicitor, Osborne Clarke, London

\title{
ICO publishes revised data protection guide
}

\author{
Ben Dunham
}

Journal of Direct, Data and Digital Marketing Practice (2015) 16, 232-233. doi:10.1057/dddmp.2015.14

Who: Information Commissioner's Office (ICO)

When: January 2015

Where: UK

Law stated as at: 4 February 2015

What happened

The Information Commissioner's Office (ICO) has recently updated its Guide to Data Protection (the 'Guide'). This has come about as part of the re-organization of the ICO website. The different sections of the Guide can now be accessed from one page of the ICO's website via the index, or can be accessed as a whole in PDF form. 
Minor additions

Social media subject
access requests (SARs)

Data transfer checklist

Model clauses for transfers

\section{Why this matters}

In terms of content relating to the data protection principles, the updated Guide generally maintains the same wording as the previous version.

Amendments to the substantive guidance are minimal, although there are a couple of additions (albeit minor) that might be of relevance to marketers holding personal information:

- The Guide now specifically acknowledges that subject access requests (requests from an individual to see what personal information about them is held by an organization) can be validly made by means of social media (as well as by email, fax or post).

- The Guide now incorporates the checklist that was previously available on the ICO website for businesses to use when considering transferring personal data overseas in order to decide whether they are compliant with the eighth data protection principle (which states that personal data shall not be transferred to a country or territory outside the European Economic Area (EEA) unless that country or territory ensures an adequate level of protection for the rights and freedoms of data subjects in relation to the processing of personal data).

- The Guide also sets out a test for assessing the adequacy of the measures for protection of personal data being transferred outside the EEA, although the test is relatively general, and with regard to ensuring that relevant safeguards are in place for transfers outside the EEA, the Guide confirms that, although the ICO has continued to authorize both the original 2001 version and the revised 2004 version of the European Commission's model clauses for data controller to data controller transfers, it only authorizes the revised 2010 clauses for data controller to data processor transfers.

Therefore, any new transfer agreements based on the original 2001 model clauses for data controller to data processor transfers will not be deemed as authorized by the ICO.

The updated Guide also provides links to further ICO guidance on certain matters. As such, the ICO has advised that the new version of the Guide be used as a replacement for previous versions.

Marketers who deal with personal data should be aware that the updated Guide is the first port of call in terms of the UK regulator's views on how to comply with data protection laws. Although the content has not changed dramatically when compared with the previous version, the updated Guide should be used from this point on as it contains a few additional compliance considerations and provides links to the most recent additional ICO guidance on specific topics.

Ben Dunham, Associate, Osborne Clarke, London 